\title{
Simulation of Motor Impairment in Head-Controlled Pointer Fitts' Law Task
}

\author{
Syed Asad Rizvi, Ella Tuson, Breanna Desrochers, John Magee \\ Clark University, Math and Computer Science Department \\ Worcester, MA 01610, USA \\ \{asrizvi,btuson,bdesrochers,jmagee\}@ clarku.edu
}

\begin{abstract}
Participants with motor impairments may not always be available for research or software development testing. To address this, we propose simulation of users with motor impairments interacting with a head-controlled mouse pointer system. Simulation can be used as a stand-in for research participants in preliminary experiments and can serve to raise awareness about ability-based interactions to a wider software development population. We evaluated our prototype system using a Fitts' Law experiment and report on the measured communication rate of our system compared to users without motor impairments and with a previously reported participant with motor impairments.
\end{abstract}

\section{CCS Concepts}

-Human-centered computing $\rightarrow$ Empirical studies in accessibility; Accessibility systems and tools;

\section{Author Keywords}

Accessibility; Head-Controlled Mouse Pointer; Camera

Mouse; Motor Impairments; Simulation; Fitts' Law

\section{INTRODUCTION}

Improving accessibility software for people with motor impairments (MI) is challenging because researchers and software developers may not have consistent access to participants with disabilities who can test the software. Furthermore, mainstream user interface (UI) developers may have a lack of awareness about diverse user needs, or when they are aware, they are unsure of how to design or test their interfaces to work for people of all abilities.

Recent work proposes to address similar challenges for people with color blindness (Impaired Color Vision) through the use of simulations [9]. The simulations allow a person to see what a color-blind individual would see and can be used both to raise awareness and to improve the use of color in designs.

Permission to make digital or hard copies of part or all of this work for personal or classroom use is granted without fee provided that copies are not made or distributed for profit or commercial advantage and that copies bear this notice and the full citation on the first page. Copyrights for third-party components of this work must be honored. For all other uses, contact the owner/author(s).

ASSETS 2018, October 22-24, 2018, Galway, Ireland

Copyright is held by the author/owner(s)

ACM ISBN 978-1-4503-5650-3/18/10.

https://doi.org/10.1145/3234695.3241034
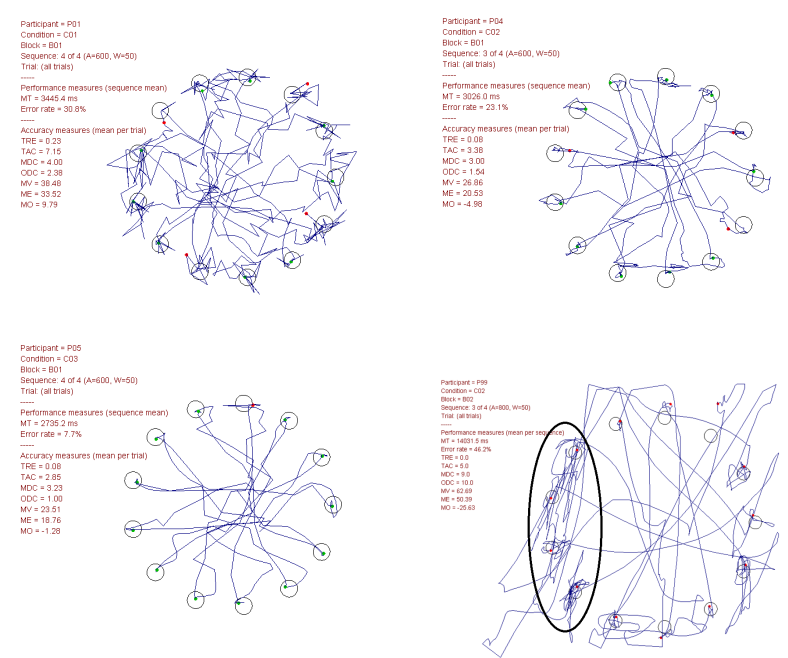

Figure 1. Mouse traces from Fitts' Law task. Top traces depict our simulation conditions $\mathrm{C} 1$ and $\mathrm{C2}$. Bottom-left depicts non-MI user with no simulation baseline (C3). Bottom-right shows trace of a participant with MI in a previous study.

In this paper, we propose to simulate a user MI using the Camera Mouse $^{1}$ [1], a computer-vision-based mouse-replacement interface that tracks head motion to move a mouse pointer on the screen.

Access to research participants with MI is challenging; partnerships with organizations in the community are necessary to access a pool of participants that are representative of the target user population $[4,3,16,17]$. Simulations may help reduce the burden on participants with motor impairments, especially at early stages of research or software development [15].

We extend prior work intended to adapt to an individual's motion abilities in controlling a mouse pointer with their head [12, 13]. The previous work is an ability-based interaction [21] technique for a user with MI. Here, the adaptations work the opposite way: we intend to simulate the abilities of user with motor impairments for a user with typical motor control.

We conducted an evaluation using Fitts' law (Fig. 1), a widely used standard for evaluating pointing devices $[10,11,18]$.

\footnotetext{
${ }^{1}$ The Camera Mouse is freely available as a download at http://www. cameramouse.org/
} 


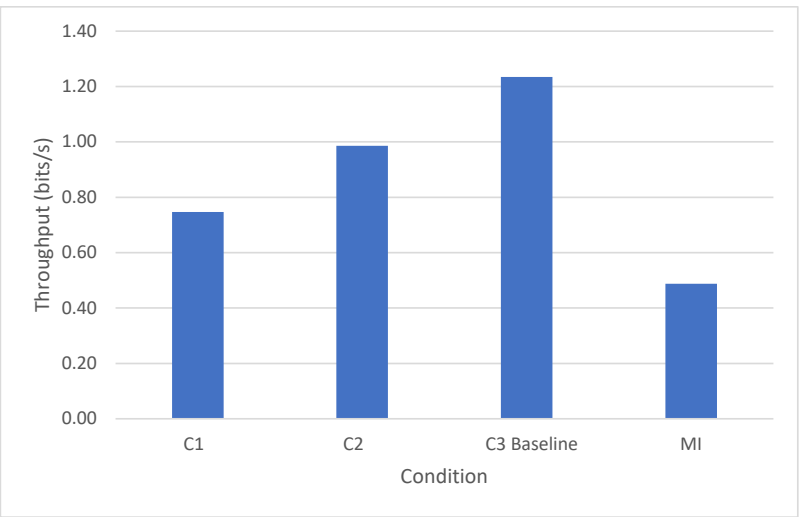

Figure 2. Throughput (bits/s). Left two bars show our simulation conditions. Third bar shows non-MI users with normal condition. Fourth bar shows MI user previously reported.

A key benefit of this evaluation metric is that it allows crossstudy comparison of a throughput measure, or "rate of communication" in bits/s. We compare our results in this paper to previously obtained measures for an individual with a motor neuron disease and for a group of participants without disabilities [14]. The results show reasonable progress towards a simulation of the user with MI.

Various approaches to improving pointer control accessibility (e.g. [18, 7] ) and clicking techniques (e.g. [19, 20, 5]) have been proposed. The performance of users with and without motor impairments $[6,8]$ is also a topic of interest. Accessibility simulations have been proposed within other interaction domains (e.g. [2, 15]).

\section{METHODS}

\section{Simulation by Additive Noise}

We developed two algorithms that affect how the Camera Mouse moves the mouse pointer. The first algorithm (C1) is characterized by a $30 \%$ probability that random movement ("noise") will be added to the pointer upon movement. The noise added by this algorithm randomly moves the pointer up to 50 pixels in both the $X$ and $Y$ directions. This algorithm was developed first, based on the hypothesis that adding random noise to the cursor when it is moved will decrease the throughput of a user with no motor impairments, resulting in a performance on the Fitts' Law evaluation like that of an individual with $\mathrm{MI}$.

The second algorithm (C2) was developed as an attempt to refine $\mathrm{C} 1$ by including a threshold for the movement of the cursor below which less noise is added. When pointer movement is slow (than 15 pixels), additive noise is reduced from $+/-50$ to between -10 and 10 , resulting in less dramatic aberrations from the user's intended path.

\section{Participants and Apparatus}

We performed an evaluation of the previously discussed algorithms to simulate a MI user. Four participants $(M=21)$, two male and two female, without motor impairments participated in the experiment.
The experiment was conducted on a 15-inch laptop screen at a resolution of $1920 \times 1080$, from a distance of approximately 2.5 feet. The following Camera Mouse settings were used: medium horizontal and vertical gain, 1.0 second dwell-time, very low smoothing, and "Normal" dwell-time click area.

\section{Procedure and Design}

We utilized an interactive evaluation tool called FittsTaskTwo ${ }^{2}$ [10]. Participants move the mouse pointer to a target and click on it before moving to the next one. The mouse trajectories and click data for each participant was stored in a log file for analysis. Each participant session consisted of four sequences of thirteen targets at amplitudes of 300 and 600 with widths of 50 and 80 pixels. The independent variable was the algorithm, described previously, with the following three conditions: $\mathrm{C} 1$ probability-based algorithm; C2 - probability with threshold limit; C3 - baseline Camera Mouse settings. This results in 156 trials ( 3 x 4 x 13) for each participant. Dependent variables are movement time (speed), throughput (bits/s), error rate (\%), and target re-entries.

\section{RESULTS}

The mean throughput of each condition is shown in Fig. 2. The baseline condition (C3) throughput rate averaged 1.23 bits/s, which is consistent with previously reported results of $1.28 \mathrm{bit} / \mathrm{s}$ for non-MI users [14].

$\mathrm{C} 1$ achieved an average throughput closest to the individual with motor impairment. The mean movement time, throughput, error rate, and target re-entries for $\mathrm{C} 1$ were $3163.97 \mathrm{~ms}$, $31.7 \%, 0.747$ bits/s and 0.173 , respectively. The throughput rate of $0.747 \mathrm{bits} / \mathrm{s}$ is still higher than the throughput reported of $0.488 \mathrm{bits} / \mathrm{s}$ of the MI participant in the previous study, but significantly lower than the baseline throughput. C2's throughput (0.98 bits/s) was higher than $\mathrm{C} 1$, and was approximately twice that of the MI participant.

\section{DISCUSSION AND CONCLUSION}

In this paper, we presented two algorithms and evaluated their effectiveness at simulating a user with motor impairments. Consistent random noise appears to be the more accurate simulation of the individual with MI based on throughput. However, the traces for the cursor movement in Fig. 1 show that the simulated trajectories could still be improved to more closely resemble the trace for the user with MI. While the trace for C2 appears closer to that of the MI individual, comparison of the measured throughputs indicates that $\mathrm{C} 1$ was a more accurate simulation with regard to rate of communication. For future work, we plan to apply machine learning techniques to better simulate the mouse traces and communication rate for the individual with motor impairments.

\section{ACKNOWLEDGMENTS}

NSF support for this project is acknowledged and greatly appreciated (\#IIS-1551590). The authors wish to thank Rashif Al-Mahmood for assistance with experiments.

\footnotetext{
${ }^{2}$ The software is freely available as a download at http://www. yorku.ca/mack/HCIbook/.
} 


\section{REFERENCES}

1. M. Betke, J. Gips, and P. Fleming. 2002. The Camera Mouse: Visual Tracking of Body Features to Provide Computer Access For People with Severe Disabilities. IEEE Transactions on Neural Systems and Rehabilitation Engineering 10, 1 (March 2002), 1-10.

2. P. Biswas, P. Robinson, and P. Langdon. 2012. Designing Inclusive Interfaces Through User Modeling and Simulation. International Journal of HumanâĂŞComputer Interaction 28, 1 (2012), 1-33. DOI : http://dx.doi .org/10.1080/10447318.2011.565718

3. M. Dee and V. L. Hanson. 2016a. 'Just Passing Through': Research in Care Homes. interactions 23, 5 (Aug. 2016), 58-61. DOI : http://dx. doi .org/10.1145/2968034

4. M. Dee and V. L. Hanson. 2016b. A Pool of Representative Users for Accessibility Research: Seeing Through the Eyes of the Users. ACM Trans. Access. Comput. 8, 1, Article 4 (Jan. 2016), 31 pages. DOI: http://dx.doi.org/10.1145/2845088

5. W. Feng, M. Chen, and M. Betke. 2014. Target Reverse Crossing - A Selection Method for Camera-based Mouse-replacement Systems. In The 7th International Conference on Pervasive Technologies Related to Assistive Environments (PETRA), Rhodes, Greece. 4 pages.

6. L. Findlater, J. E. Froehlich, K. Fattal, J. O. Wobbrock, and T. Dastyar. 2013. Age-related Differences in Performance with Touchscreens Compared to Traditional Mouse Input. In Proceedings of the SIGCHI Conference on Human Factors in Computing Systems (CHI'13). 343-346. DOI :

http://dx.doi.org/10.1145/2470654.2470703

7. L. Findlater, A. Jansen, K. Shinohara, M. Dixon, P. Kamb, J. Rakita, and J. O. Wobbrock. 2010. Enhanced area cursors: reducing fine pointing demands for people with motor impairments. In Proceedings of the 23nd Annual ACM Symposium on User Interface Software and Technology (UIST'10), New York, NY. 153-162.

8. L. Findlater, K. Moffatt, J. E. Froehlich, M. Malu, and J. Zhang. 2017. Comparing Touchscreen and Mouse Input Performance by People With and Without Upper Body Motor Impairments. In Proceedings of the 2017 CHI Conference on Human Factors in Computing Systems (CHI'17). 6056-6061. DOI : http://dx.doi .org/10.1145/3025453.3025603

9. R. MacAlpine and D. R. Flatla. 2016. Real-Time Mobile Personalized Simulations of Impaired Colour Vision. In Proceedings of the 18th International ACM SIGACCESS Conference on Computers and Accessibility (ASSETS '16). ACM, New York, NY, USA, 181-189. DOI : http://dx.doi .org/10.1145/2982142.2982170

10. I. S. MacKenzie. 2013. Human-Computer Interaction: An Empirical Research Perspective (1st ed.). Morgan Kaufmann Publishers Inc., San Francisco, CA, USA.
11. I. S. MacKenzie. 2018. Fitts' law. In Handbook of human-computer interaction, J. Kirakowski K. L. Norman (Ed.). Wiley, Hoboken, NJ, Chapter 17, 349-370.

12. J. J. Magee, S. Epstein, E. S. Missimer, and M. Betke. 2010. Adaptive Mappings for Mouse-replacement Interfaces. In Proceedings of the 12th International ACM SIGACCESS Conference on Computers and Accessibility (ASSETS '10). ACM, New York, NY, USA, 231-232. DOI : http://dx.doi.org/10.1145/1878803.1878846

13. J. J. Magee, S. Epstein, E. S. Missimer, C. Kwan, and M. Betke. 2011. Adaptive mouse-replacement interface control functions for users with disabilities. In Proc. HCI International 2011, UAHCI/HCII 2011, LNCS 6766. Springer, 332-341.

14. J. J. Magee, T. Felzer, and I. S. MacKenzie. 2015. Camera Mouse + ClickerAID: Dwell vs. Single-Muscle Click Actuation in Mouse-Replacement Interfaces. In Proc. HCI International 2015, UAHCI/HCII 2015, LNCS 9175. Springer, 74-84.

15. J. Mankoff, H. Fait, and R. Juang. 2005. Evaluating accessibility by simulating the experiences of users with vision or motor impairments. IBM Systems Journal 44, 3 (2005), 505-517. DOI :

http://dx.doi.org/10.1147/sj .443.0505

16. L. McIntyre and V. L. Hanson. 2013. BESIDE: The Built Environment for Social Inclusion in the Digital Economy. In CHI' 13 Extended Abstracts on Human Factors in Computing Systems (CHI EA '13). 289-294. DOI : http://dx.doi .org/10.1145/2468356.2468408

17. G. Webster and V. L. Hanson. 2014. Technology for Supporting Care Staff in Residential Homes. ACM Trans. Access. Comput. 5, 3, Article 8 (Jan. 2014), 23 pages. DOI : http://dx.doi .org/10.1145/2543577

18. J. O. Wobbrock, J. Fogarty, S.-Y. Liu, S. Kimuro, and S. Harada. 2009. The angle mouse: Target-agnostic dynamic gain adjustment based on angular deviation. In Proceedings of the 27th International Conference on Human Factors in Computing Systems (CHI '09), Boston, MA. 1401-1410.

19. J. O. Wobbrock and K. Z. Gajos. 2007. A comparison of area pointing and goal crossing for people with and without motor impairments. In Proceedings of the ACM SIGACCESS Conference on Computers and Accessibility (Assets'07). ACM Press, New York, NY, USA, 3-10.

20. J. O. Wobbrock and K. Z. Gajos. 2008. Goal Crossing with Mice and Trackballs for People with Motor Impairments: Performance, Submovements, and Design Directions. ACM Transactions on Accessible Computing 1, 1 (2008), 1-37.

21. J. O. Wobbrock, K. Z. Gajos, S. K. Kane, and G. C. Vanderheiden. 2018. Ability-based Design. Commun. ACM 61, 6 (May 2018), 62-71. DOI: http://dx.doi.org/10.1145/3148051 